\title{
Ultrassonografia Intervencionista para implantação e monitoramento de cateter venoso central de inserção periférica: scoping review
}

\author{
Interventional ultrasound for implantation and monitoring of peripherally inserted central venous catheter: \\ scoping review
}

\author{
Ultrasonido intervencionista para implantación y monitorización de catéter venoso central insertado \\ periféricamente: revisión del alcance
}

\author{
Andréa Maria Alves Vilar'; Márcia Farias de Oliveira"l; Corina Maria Mattos ${ }^{\prime \prime \prime}$; Zenith Rosa Silvino ${ }^{\prime V}$
}

\begin{abstract}
RESUMO
Objetivo: discutir as evidências disponíveis sobre uso de ultrassonografia na implantação/manutenção de cateter venoso central de inserção periférica no neonato crítico. Método: scoping review, baseada nas recomendações de especialistas do Joanna Briggs Institute. Para identificar a questão de investigação foi seguida a versão PCC - Population, Concept and Context. As buscas foram realizadas entre janeiro e março de 2020, em três bases dados e no Google Scholar. Resultados: das 354 publicações encontradas resultou uma amostra de 15 artigos, publicados em diversos países, entre 2016 e 2020, em inglês e português. A maioria evidenciava uso da ultrasound point of care (POCUS) para escolha do sítio venoso ou localização da ponta do cateter. Conclusão: são necessários mais estudos, investigando a efetividade da POCUS na inserção/manutenção de cateter epicutâneo em neonatos críticos, para basear sua adoção como padrão ouro nesta clientela. O manejo por enfermeiros ainda é incipiente. Descritores: Recém-Nascido; Unidades de Terapia Intensiva Neonatal; Ultrassonografia de Intervenção; Cateteres.
\end{abstract}

\begin{abstract}
Objective: to discuss the available evidence on the use of ultrasound in insertion and maintenance of peripherally inserted central venous catheters in critical neonates. Method: this scoping review was based on recommendations by experts from the Joanna Briggs Institute. The population, concept and context (PCC) approach was used to identify the research question. The study was carried out between January and March 2020 in three databases and Google Scholar. Results: a sample of 15 articles published in several countries between 2016 and 2020, in English and Portuguese, was obtained in the 354 publications found. Most showed ultrasound point of care (POCUS) being used to choose the venous site or locate the catheter tip. Conclusion: further studies are needed to investigate the effectiveness of POCUS in insertion and maintenance of epicutaneous catheters in critical neonates, to support its adoption as the gold standard in this clientele. Handling by nurses is still incipient. Descriptors: Infant, Newborn; Intensive Care Units, Neonatal; Ultrasonography, Interventional; Catheters.
\end{abstract}

\section{RESUMEN}

Objetivo: discutir la evidencia disponible sobre el uso de el ultrasonido en la inserción y mantenimiento de catéteres venosos centrales de inserción periférica en neonatos críticos. Método: esta revisión de alcance se basó en recomendaciones de expertos del Instituto Joanna Briggs. Se utilizó el enfoque de población, concepto y contexto (PCC) para identificar la pregunta de investigación. El estudio se realizó entre enero y marzo de 2020 en tres bases de datos y Google Scholar. Resultados: en las 354 publicaciones encontradas se obtuvo una muestra de 15 artículos publicados en varios países entre 2016 y 2020, en inglés y portugués. La mayoría mostró que se usaba el punto de atención de ultrasonido (POCUS) para elegir el sitio venoso o ubicar la punta del catéter. Conclusión: se necesitan más estudios para investigar la efectividad de POCUS en la inserción y mantenimiento de catéteres epicutáneos en neonatos críticos, para respaldar su adopción como el estándar de oro en esta clientela. El manejo por parte de enfermeras es aún incipiente.

Descriptores: Recién Nacido; Unidades de Cuidado Intensivo Neonatal; Ultrasonografia Intervencional; Catéteres.

\section{INTRODUÇÃO}

No cuidado ao recém-nascido (RN) internado em Unidades de Terapia Intensiva Neonatal (UTIN), grandes avanços tecnológicos na área das ciências da saúde, somados à prestação de assistência multiprofissional de qualidade, trouxeram considerável crescimento na taxa de sobrevivência desta clientela, mesmo entre prematuros extremos.

'Enfermeira. Mestre. Intensivista Neonatal. Membro do Núcleo de Estudos e Pesquisa em Cidadania e Gerência na Enfermagem (NEGICEN). Universidade do Estado do Rio de Janeiro. Brasil. E-mail: andreavilar72@yahoo.com.br. ORDIC: https://orcid.org/0000-0001-8321-5397

"Enfermeira. Mestre. Intensivista Neonatal. Membro do Núcleo de Estudos e Pesquisa em Cidadania e Gerência na Enfermagem (NEGICEN). Universidade do Estado do Rio de Janeiro. Brasil. E-mail: marcia_red@ig.com.br. ORCID: https://orcid.org/0000-0002-1804-8833

I'Enfermeira. Mestre. Hospital Infantil Ismélia da Silveira e da Unidade de Pronto Atendimento Walter Garcia. Membro do Núcleo de Estudos e Pesquisa em Cidadania e Gerência na Enfermagem. Universidade Federal Fluminense, Niterói, Brasil. E-mail: coramary@gmail.com. ORCID: https://orcid.org/0000-0002-7093-682X

IVEnfermeira. Doutora. Professora Titular. Coordenadora do Núcleo de Estudos e Pesquisa em Cidadania em Gerência na Enfermagem. Universidade Federal Fluminense. Niterói, Brasil. E-mail: zenithrosa52@gmail.com. ORCID: https://orcid.org/0000-0002-2848-9747 
A modernização das UTIN, bem como o aprimoramento técnico-científico no manejo do RN de alto risco, propiciaram um salto na qualidade da assistência. A terapia intravenosa (TIV), indispensável para a manutenção da vida do neonato crítico, é um dos procedimentos que espelha este avanço tecnológico. Cateteres biocompatíveis, fixadores, curativos industriais e dispositivos, utilizados na seleção adequada do vaso sanguíneo, foram desenvolvidos recentemente, e maximizaram os resultados terapêuticos, com maior relação custo/benefício ${ }^{1,2}$.

Para a realização da TIV, é fundamental a viabilização de acesso venoso confiável, utilizando dispositivos como cateter de teflon sobre agulha, cateter umbilical, cateter venoso central de inserção periférica (CCIP) e cateter venoso central $^{1}$. CCIP são dispositivos confeccionados em silicone e poliuretano (materiais de baixa trombogenicidade, biocompatíveis e bioestáveis), introduzidos por veia superficial, de extremidade superior ou inferior, até atingirem o terço distal da veia cava superior ou o terço proximal da veia cava inferior, conferindo assim características de acesso central. São, na atualidade, os dispositivos de longa permanência mais utilizados para possibilitar TIV em Neonatologia.

Sua implantação é um procedimento eletivo, geralmente programado para substituir o cateterismo umbilical venoso ou acesso periférico. É indicado em condições tais como monitoramento hemodinâmico, infusão de soluções hiperosmolares, como nutrição parenteral, uso de medicamentos irritantes/vesicantes, além de antibioticoterapia superior a seis dias. Prematuros extremos que recebem nutrição por meio de cateter epicutâneo têm ganho de peso significativo, menor tempo de internação e menores taxas de infecção, quando comparados com bebês submetidos à múltiplas punções periféricas ${ }^{2-4}$.

Porém, estabelecer acesso venoso seguro e durável, via periferia, pode ser desafiador, quando a clientela é o RN crítico. Cada vaso apresentará dificuldades, riscos e complicações particulares. E todos terão diminuto diâmetro. Neste contexto, acessar uma via central utilizando cateter epicutâneo, com técnica asséptica rigorosa e adoção de melhores práticas, reduz eventos adversos, contribuindo para a segurança do paciente. A orientação por ultrassom (USG), usado para a colocação do CCIP (ou PICC, acrônimo da locução em inglês peripherally inserted central catheter) melhora a precisão da colocação, reduzindo o tempo de inserção, taxas de insucesso e complicações relacionadas a este dispositivo ${ }^{5}$, e auxilia na redução da exposição à radiação, já que há indícios que o monitoramento radiográfico deste cateter contribui muito para a exposição dos RN, com efeito deletério. Há relatos que RN de muito baixo peso ao nascer são submetidos, em média, a 25 radiografias durante o período de permanência em UTIN. A adoção de protocolos institucionais de inserção que preconizem o uso de USG é, portanto, desejável ${ }^{6}$.

Para a legislação brasileira, profissionais médicos são responsáveis legais pelas técnicas cirúrgicas de cateterismo venoso central. No âmbito da equipe de enfermagem, cateterismo umbilical e PICC são privativos do enfermeiro, desde que submetido à qualificação e/ou capacitação profissional ${ }^{3}$.

Para prevenir complicações, estimadas em até 33\%, a habilidade de inserir é fundamental ${ }^{7}$. Segundo diretrizes da Infusion Nursing Society (INS) do Brasil, a formação de uma equipe de trabalho, capacitada e estruturada, um time dedicado exclusivamente à TIV, torna realidade o trabalho com excelência, assegurando as melhores práticas. A garantia da educação continuada para profissionais e estudantes, em relação à instalação de acessos vasculares, é outro ganho citado ${ }^{8}$.

Em 2019, o Serviço de Enfermagem de um Hospital Universitário do Estado do Rio de Janeiro, iniciou trabalhos para a implantação do time de cateter, objetivando adotar as melhores práticas em cateterismos venosos, visando à qualidade da assistência, valorando a responsabilidade com a segurança do paciente. O objetivo estratégico traçado foi padronizar a inserção, fixação, manutenção e retirada de acessos vasculares. Iniciados os trabalhos, lideranças setoriais foram estabelecidas. Para o alcance das tecnologias que refletissem a excelência na área, o estado da arte foi realizado.

Este artigo é o relato de um estudo de revisão de escopo proposto para discutir evidências disponíveis sobre uso de USG na implantação/manutenção de PICC no neonato crítico. Mais especificamente buscamos responder à seguinte questão norteadora: quais são as evidências científicas atuais sobre o uso da USG na implantação e manutenção do PICC no neonato crítico?

\section{MÉTODO}

O estudo foi delineado como revisão de escopo (scoping review), em conformidade com método estruturado pelo Instituto Joanna Briggs ${ }^{9}$, que possibilita mapear conceitos fundamentais, aclarar áreas de pesquisa e identificar lacunas do conhecimento ${ }^{10}$. Como em outras metodologias de revisão de literatura, a busca é norteada pela escolha da pergunta de pesquisa. E os resultados encontrados filtrados por aplicação de critérios de inclusão e exclusão. Porém esta inclusão/exclusão não é pautada pela qualidade dos trabalhos (posto que essa avaliação não faz parte do objetivo da scoping review), mas pela relevância na discussão do tema. Os dados selecionados representam uma síntese essencialmente qualitativa ${ }^{11}$.

Outra particularidade do método é a discussão dos resultados, e não análise, que pode ser realizada por especialistas na área, buscando a contextualização e síntese de produções, disseminando o conhecimento ${ }^{11}$. 
Para construção da pergunta de pesquisa, utilizou-se, em janeiro de 2020 a estratégia Population, Concept e Context $(\mathrm{PCC})^{9}$, sendo $\mathrm{P}$ o tipo de população (recém-nascido), C o conceito (implantação e manutenção do cateter venoso central de inserção periférica com uso de USG), e C o contexto (unidade de terapia intensiva neonatal).

Esta etapa inicial do protocolo serviu para identificar a questão de pesquisa e construir as estratégias de busca. Pretendeu-se responder à questão norteadora: quais as evidências científicas disponíveis sobre o uso da USG na implantação e manutenção do PICC em RN críticos?

A seleção dos artigos foi realizada de janeiro a março de 2020, por dois pesquisadores, de forma independente. Pelo Portal Biblioteca Virtual em Saúde (BVS) foram consultadas as bases de dados LILACS e IBECS. A interface National Library of Medicine (PubMed) serviu de acesso ao seu principal componente, a Medical Literature Analysis and Retrievel System Online (MEDLINE). A biblioteca eletrônica Scientific Eletronic Library Online (SciELO) foi o último portal eletrônico a compor esta fase de busca. Os descritores selecionados foram "newborn", "catheter", "catheterization", "ultrassonography" e "cannulation" e suas variações em espanhol e português, além de termos livres relacionados/sinônimos, como PICC ou CCIP. Para extração dos descritores foram consultados os vocabulários controlados da área da saúde DeCs (Descritores em Ciências da Saúde) e MeSH (Medical Subject Headings). Para a correlação adequada dos termos, foram utilizados os operadores booleanos "AND" e "OR".

Os títulos incluídos na revisão atenderam aos seguintes critérios: ser publicado entre o período de fevereiro de 2015 e fevereiro de 2020; em periódicos nacionais e internacionais; estar disponíveis nos idiomas português, inglês ou espanhol, que respondessem à questão norteadora. Optou-se por estabelecer um recorte temporal dos últimos cinco anos (2015 a 2020), para recuperar publicações mais recentes sobre a temática abordada. Foram excluídas publicações em duplas e textos não vinculados à temática. Esta estratégia de busca e o quantitativo obtido em cada base de dados podem ser observados na Figura 1.

\begin{tabular}{|c|c|c|c|}
\hline BASE & ESTRATÉGIA DE BUSCA & \multicolumn{2}{|l|}{ QUANTITATIVO } \\
\hline \multirow{6}{*}{$\sum_{\infty}^{n}$} & \multirow{6}{*}{$\begin{array}{l}\text { tw:(Newborn OR "recem-nascido" OR "recien nacido" OR neonato* } \\
\text { OR neonata* OR neonate*)) AND (tw:(Catheter* OR cateter* OR } \\
\text { PICC OR CCIP OR canula OR cannulation OR cannula)) AND } \\
\text { (tw:(Ultrasonography OR ultrassonografia OR ultrassom OR } \\
\text { ultrasonografia OR ultrasonido OR ultra-som OR ecografia)) AND } \\
\text { (instance:"regional") AND (db:("LILACS" OR "IBECS") AND } \\
\text { year_cluster:("2015" OR "2016" OR "2017" OR "2018" OR "2019")) }\end{array}$} & Identificados & 23 \\
\hline & & Excluídos pelo título & 13 \\
\hline & & Duplicatas & 01 \\
\hline & & $\begin{array}{l}\text { Selecionados para leitura dos } \\
\text { resumos }\end{array}$ & 09 \\
\hline & & $\begin{array}{l}\text { Selecionado para leitura de texto } \\
\text { completo }\end{array}$ & 04 \\
\hline & & Selecionado para discussão & 01 \\
\hline \multirow{6}{*}{ 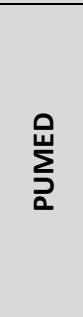 } & \multirow{6}{*}{$\begin{array}{l}\text { ((Newborn[mh] OR Newborn[tiab]) AND ((Catheters[mh] OR } \\
\text { catheter*[tiab] OR PICC[tiab] OR cannula[tiab] OR cannulation[tiab]) } \\
\text { AND (Ultrasonography[mh] OR Ultrasonography[tiab] OR ultra- } \\
\text { sound[tiab]))) AND ("2015")/01/09"[PDat]) [PDat] : } \\
\text { "2020/01/06"[PDat]) }\end{array}$} & Identificados & 261 \\
\hline & & Excluídos pelo título & 210 \\
\hline & & Duplicatas & 06 \\
\hline & & $\begin{array}{l}\text { Selecionados para leitura do } \\
\text { resumo }\end{array}$ & 45 \\
\hline & & $\begin{array}{l}\text { Selecionados para leitura de } \\
\text { texto completo }\end{array}$ & 10 \\
\hline & & Selecionados para discussão & 09 \\
\hline \multirow{6}{*}{ 号 } & \multirow{6}{*}{$\begin{array}{l}\text { newborn AND catheter* AND ultrasonography OR ultra-som AND } \\
\text { year_cluster:("2015" OR "2016" OR "2017" OR "2018" OR "2019") }\end{array}$} & Identificados & 35 \\
\hline & & Excluídos pelo título & 27 \\
\hline & & Duplicatas & 03 \\
\hline & & $\begin{array}{l}\text { Selecionados para leitura do } \\
\text { resumo }\end{array}$ & 05 \\
\hline & & $\begin{array}{l}\text { Selecionados para leitura de texto } \\
\text { completo }\end{array}$ & 0 \\
\hline & & Selecionados para discussão & 0 \\
\hline
\end{tabular}

FIGURA 1: Protocolo de busca e seleção de artigos em bases de dados

Em relação à Grey Literature, foi realizada uma busca, no Google Scholar, por títulos identificados em referências de estudos capturados ou com uso de palavras-chaves, obedecendo os mesmos critérios da busca nas bases de dados PubMed.

Todos os dados foram extraídos por duas autoras e confirmados por uma terceira, experiente em Scoping Review. A síntese dos resultados foi realizada pelas quatro autoras, de forma consensual. 


\section{RESULTADOS}

Dos 319 estudos identificados nas bases de dados, após leitura exaustiva de seus títulos e resumos, 14 foram selecionados, por preencherem os critérios de inclusão.

Entre os eleitos, quatro foram exclusos por não contemplarem totalmente a temática, se referindo apenas a outros cateteres venosos centrais que não PICC ou população diversa de RN críticos. Os 10 estudos restantes foram analisados e inclusos na pesquisa.

As buscas no Google Scholar resultaram em acréscimo de 35 publicações. Após leitura de resumos e/ou texto completo foram eleitos cinco documentos, dois pela busca com palavras-chave e três encontrados em referências de outros estudos.

Ao final, a amostra desta revisão totalizou em 15 escolhidos para discussão. $O$ processo integral de busca e seleção dos estudos desta revisão está representado em fluxograma (Figura 2), conforme recomendações do JBI, segundo checklist adaptado do Preferred Reporting Items for Systematic reviews and Meta-Analyses extension for Scoping Reviews (PRISMA-ScR) ${ }^{9}$.

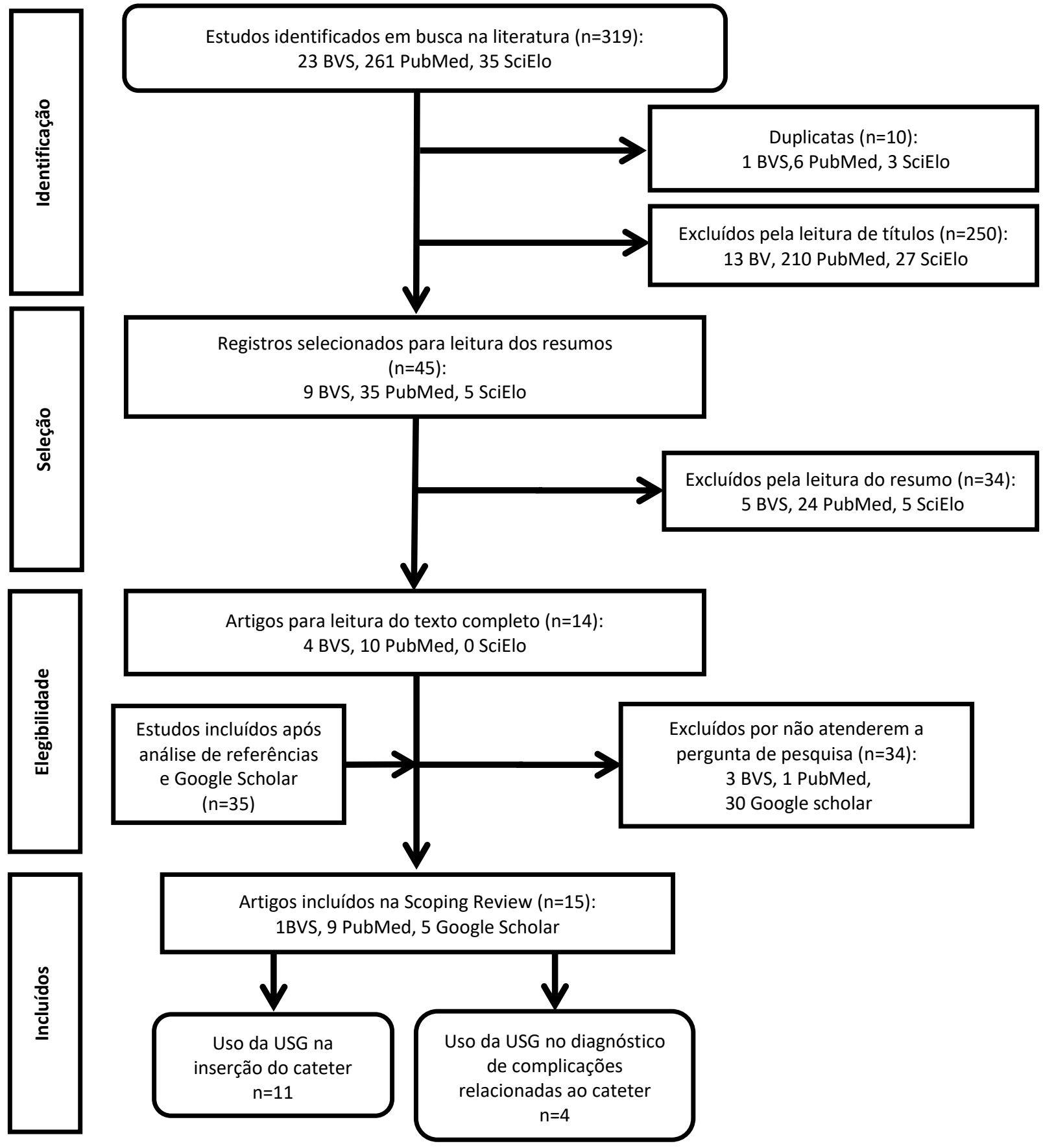

Figura 2: Fluxograma: processo integral de busca e seleção de publicações. Niterói, RJ, Brasil, 2020. 
Os quinze estudos incluídos foram publicados entre 2016 a 2020 (Figuras 3 e 4). Não houve aproveitamento de estudos de 2015. Em 2016, ao contrário, houve forte concentração, com seis estudos publicados ${ }^{19-24}$, seguido do ano de 2018, com quatro ${ }^{15-18}$. Em relação ao local de desenvolvimento das pesquisas, a Europa ${ }^{12}$ foi cenário de um estudo, assim como Eurásia ${ }^{16}$, Ásia ${ }^{20}$ e Oceania ${ }^{26}$. Nas Américas foram desenvolvidos onze, sendo oito estadunidenses ${ }^{13,14,18,19,21-}$ ${ }^{24}$ e três brasileiros ${ }^{15,17,25}$. Três documentos foram disponibilizados em português ${ }^{15,17,25}$, os demais em inglês ${ }^{12-14,16,18-24,26}$.

\begin{tabular}{|c|c|c|c|c|}
\hline $\begin{array}{c}\text { Autor } \\
\text { Local/Base/Ano }\end{array}$ & Objetivos & $\begin{array}{c}\text { Desenho do } \\
\text { estudo/Amostra }\end{array}$ & Principais Resultados & Conclusão \\
\hline $\begin{array}{l}\text { Singh, et al }{ }^{12} \\
\text { Reino Unido } \\
\text { Google Scholar } \\
2020\end{array}$ & $\begin{array}{l}\text { Fornecer diretrizes } \\
\text { baseadas em evidências } \\
\text { para o uso de POCUS } \\
\text { (ultrassom no local de } \\
\text { atendimento, em inglês) } \\
\text { em RN e crianças } \\
\text { gravemente enfermos. }\end{array}$ & $\begin{array}{l}\text { Revisão } \\
\text { sistemática }\end{array}$ & $\begin{array}{l}\text { Os especialistas concordaram com } 39 \text { das } 41 \\
\text { recomendações para o uso de POCUS em RN } \\
\text { críticos. Em relação a POCUS vascular: para guiar } \\
\text { PICC, resulta em maior taxa de canulação na } \\
\text { primeira tentativa quando comparada à técnica de } \\
\text { referência anatômica; útil para localizar a ponta do } \\
\text { cateter em RN e crianças; pode diminuir a } \\
\text { exposição ao raio-X e manipulação da linha, se } \\
\text { utilizada para confirmar a posição da ponta do } \\
\text { PICC, pós colocação. }\end{array}$ & $\begin{array}{l}\text { As diretrizes baseadas em evidências } \\
\text { para o uso de POCUS em neonatos e } \\
\text { crianças gravemente enfermos } \\
\text { estabelecidas serão uteis para } \\
\text { otimizar o uso da técnica, programas } \\
\text { de treinamento e pesquisas } \\
\text { adicionais, que são urgentemente } \\
\text { necessárias, dada a fraca qualidade } \\
\text { das evidências disponíveis. }\end{array}$ \\
\hline $\begin{array}{l}\text { Suell J, et al }\left.\right|^{13} \\
\text { Estados Unidos } \\
\text { PUBMED } \\
2020\end{array}$ & $\begin{array}{l}\text { Descrever a experiência } \\
\text { de acompanhamento } \\
\text { em tempo real (TR) de } \\
\text { localização de ponta de } \\
\text { cateter PICC com uso de } \\
\text { USG, em um RN de } 2 \mathrm{~kg} \text {, } \\
\text { cardiopata, submetido à } \\
\text { técnica de inserção às } \\
\text { cegas, por medida } \\
\text { anatômica padrão. }\end{array}$ & $\begin{array}{l}\text { Estudo de caso, } \\
\text { qualitativo }\end{array}$ & $\begin{array}{l}\text { Tanto a USG em TR quanto o raio-X identificaram o } \\
\text { mau posicionamento da ponta do cateter, } \\
\text { direcionada para a veia jugular interna esquerda. } \\
\text { Realizada a tentativa de reposicionamento guiada } \\
\text { pelo USG, utilizando a mesma medida prévia. O } \\
\text { procedimento foi encerrado quando a imagem } \\
\text { ecográfica evidenciou a ponta superior do } \\
\text { dispositivo na veia braquiocefálica esquerda, } \\
\text { confirmada por raio-X. }\end{array}$ & $\begin{array}{l}\text { O uso da USG em tempo real } \\
\text { possibilitou evidenciar o } \\
\text { posicionamento anômalo do PICC, e } \\
\text { manipulação de extremidade, } \\
\text { resultando em um melhor } \\
\text { posicionamento da linha central. }\end{array}$ \\
\hline $\begin{array}{l}\text { Motz P, et al }{ }^{14} \\
\text { Estados Unidos } \\
\text { PUBMED } \\
2019\end{array}$ & $\begin{array}{l}\text { Avaliar a viabilidade, } \\
\text { para uso de radiologistas } \\
\text { experientes de um } \\
\text { hospital de Seattle, de } \\
\text { protocolo para } \\
\text { localização da ponta de } \\
\text { PICC inseridos em RN. } \\
\end{array}$ & $\begin{array}{l}\text { Estudo piloto, } \\
\text { coorte } \\
\text { intervencionista } \\
\text { prospectivo, } \\
\text { quantitativo }\end{array}$ & $\begin{array}{l}\text { Foram realizadas } 14 \text { varreduras, em } 11 \text { neonatos, } \\
\text { dos quais oito tinham peso inferior a } 1,5 \mathrm{~kg} \text {. Um } \\
\text { raio-X de tórax foi realizado antes da USG e seu } \\
\text { resultado não era repassado ao radiologista } \\
\text { responsável pela USG. A localização do PICC, pela } \\
\text { USG foi idêntica ao raio-X em todas as varreduras } \\
\text { realizadas. }\end{array}$ & $\begin{array}{l}\text { O protocolo testado mostrou-se } \\
\text { viável para uso em RN, mas para ser } \\
\text { adotado na UTI neonatal seria } \\
\text { necessária a continuidade do } \\
\text { estudo, envolvendo uma amostra } \\
\text { maior, mais significativa. }\end{array}$ \\
\hline $\begin{array}{l}\text { Barreiros L, et al }{ }^{15} \\
\text { Brasil } \\
\text { BVS } \\
2018\end{array}$ & $\begin{array}{l}\text { Determinar a incidência } \\
\text { de derrame pericárdico } \\
\text { e tamponamento } \\
\text { cardíaco em RN de UTIN, } \\
\text { com ênfase na relação } \\
\text { entre o PICC e os } \\
\text { agravos; Avaliar o papel } \\
\text { da USG à beira-leito no } \\
\text { diagnóstico. }\end{array}$ & $\begin{array}{l}\text { Estudo } \\
\text { documental, } \\
\text { retrospectivo, } \\
\text { qualitativo }\end{array}$ & $\begin{array}{l}\text { Realizada avaliação retrospectiva de } 426 \\
\text { prontuários dos RN admitidos na UTIN. Os dados } \\
\text { evidenciaram } 285 \text { USG realizadas à beira-leito. } \\
\text { Encontrados } 6 \text { casos de derrame pericárdico, } \\
\text { sendo } 04 \text { com choque obstrutivo e necessidade de } \\
\text { realizar drenagem pericárdica. Todos os pacientes } \\
\text { evoluíram com melhora. A incidência de derrame } \\
\text { pericárdico foi de } 2,4 \text { casos por ano. }\end{array}$ & $\begin{array}{l}\text { A incidência de derrame pericárdico } \\
\text { é baixa em RN. Mas o diagnóstico } \\
\text { precoce é fundamental nos casos de } \\
\text { instalação abrupta, pelo risco de } \\
\text { morbimortalidade. Todos os casos } \\
\text { foram diagnosticados por USG a } \\
\text { beira-leito, validando a importância } \\
\text { deste exame no rastreio de } \\
\text { complicações. }\end{array}$ \\
\hline $\begin{array}{l}\text { Sancak S, et al }{ }^{16} \\
\text { Turquia } \\
\text { PUBMED } \\
2018\end{array}$ & $\begin{array}{l}\text { Relatar a experiência do } \\
\text { uso de USG para } \\
\text { confirmação de } \\
\text { posicionamento de PICC } \\
\text { em RN de } 33 \text { semanas } \\
\text { de IGC, inserido por } \\
\text { técnica tradicional, que } \\
\text { evolui com complicação }\end{array}$ & $\begin{array}{l}\text { Relato de caso, } \\
\text { qualitativo }\end{array}$ & $\begin{array}{l}\text { O controle de posicionamento pós punção, } \\
\text { realizado com raio-X não contrastado, evidenciou } \\
\text { cateter em posição periférica. Após piora clínica, foi } \\
\text { realizado USG, com visualização do cateter em } \\
\text { artéria pulmonar esquerda e líquido em pulmão } \\
\text { esquerdo (nutrição parenteral). }\end{array}$ & $\begin{array}{l}\text { Utilizar USG para confirmação da } \\
\text { posição do PICC pode trazer } \\
\text { benefícios em relação ao uso de } \\
\text { raio-X por evitar exposição à } \\
\text { radiação, podendo ser utilizado em } \\
\text { tempo real, da punção à certificação } \\
\text { da localização da ponta do cateter. }\end{array}$ \\
\hline $\begin{array}{l}\text { Torres } \mathrm{R}^{17} \\
\text { Brasil } \\
\text { Google Scholar } \\
2018\end{array}$ & $\begin{array}{l}\text { Avaliar o uso da POCUS } \\
\text { por especialista em } \\
\text { terapia intensiva no } \\
\text { auxílio diagnóstico e } \\
\text { acompanhamento de } \\
\text { crianças gravemente } \\
\text { enfermas em uma UTI } \\
\text { Neonatal }\end{array}$ & $\begin{array}{l}\text { Prospectivo, } \\
\text { Intervencionista, } \\
\text { quantitativo }\end{array}$ & $\begin{array}{l}\text { Três casos de tamponamento cardíaco relacionado } \\
\text { ao uso de PICC foram detectados, sendo } 02 \text { em } \\
\text { suspeita clínica. Em todos, o diagnóstico } \\
\text { ecocardiográfico foi fundamental para a decisão de } \\
\text { realizar a pericardiocentese. O evento foi } \\
\text { confirmado como complicação do PICC, pelas } \\
\text { características bioquímicas do liquido drenado, } \\
\text { idêntica aos líquidos infundidos. }\end{array}$ & $\begin{array}{l}\text { Os resultados evidenciaram melhor } \\
\text { performance diagnóstica quando a } \\
\text { POCUS foi utilizada como } \\
\text { complementar ao exame físico, } \\
\text { melhorando ou modificando a } \\
\text { hipótese inicial e otimizando as } \\
\text { condutas médicas. Confirmou } \\
\text { complicação correlacionada ao mau } \\
\text { posicionamento do PICC nos três } \\
\text { casos avaliados. }\end{array}$ \\
\hline $\begin{array}{l}\text { Zaghloul N, et al }^{18} \\
\text { Estados Unidos } \\
\text { Google Scholar } \\
2018\end{array}$ & $\begin{array}{l}\text { Analisar a concordância } \\
\text { entre raios-X e POCUS } \\
\text { na determinação da } \\
\text { ponta de cateteres } \\
\text { centrais; Examinar mau } \\
\text { posicionamento por } \\
\text { realização de POCUS } \\
\text { seriado. }\end{array}$ & $\begin{array}{l}\text { Observacional, } \\
\text { prospectivo, } \\
\text { quantitativo }\end{array}$ & $\begin{array}{l}\text { Avaliados } 54 \text { RN, sendo } 108 \text { "pares" de raio-X e } \\
\text { imagens POCUS. O coeficiente de concordância } \\
\text { (AC1), em relação à posição / posição incorreta da } \\
\text { ponta do CVC foi alto ( PICC-AC1 = 0,94). A taxa de } \\
\text { mau posicionamento da ponta de CVC diminuiu } \\
\text { com o tempo. }\end{array}$ & $\begin{array}{l}\text { A concordância entre os dois } \\
\text { exames para da ponta do PICC foi } \\
\text { alta, principalmente em } \mathrm{RN}<1000 \mathrm{~g} \text {. } \\
\text { Os dados sugerem POCUS como } \\
\text { eficiente para confirmação inicial e } \\
\text { acompanham da posição da ponta } \\
\text { de CVC. }\end{array}$ \\
\hline
\end{tabular}

Figura 3: Quadro Sinóptico das publicações referentes aos anos de 2018, 2019 e 2020. Niterói, RJ, Brasil, 2020. 


\begin{tabular}{|c|c|c|c|c|}
\hline $\begin{array}{c}\text { Autor } \\
\text { Local/Base/Ano }\end{array}$ & Objetivos & $\begin{array}{c}\text { Desenho do } \\
\text { estudo/Amostra }\end{array}$ & Principais Resultados & Conclusão \\
\hline $\begin{array}{l}\text { Ares Hunter }^{19} \\
\text { Estados Unidos } \\
\text { PUBMED } \\
2017\end{array}$ & $\begin{array}{l}\text { Descrever as múltiplas } \\
\text { indicações para o uso de } \\
\text { CVC e os diferentes } \\
\text { dispositivos disponíveis } \\
\text { para acesso venoso } \\
\text { central. }\end{array}$ & $\begin{array}{l}\text { Revisão } \\
\text { sistemática }\end{array}$ & $\begin{array}{l}\text { Grandes revisões sistemáticas com metanálise } \\
\text { concluíram que a orientação por USG na colocação } \\
\text { de CVC é mais segura que a técnica tradicional, } \\
\text { pelo menos para acessos em veia jugular interna. } \\
\text { Outra evidência foi a relação da colocação } \\
\text { adequada da ponta do cateter com diminuição de } \\
\text { complicações. }\end{array}$ & $\begin{array}{l}\text { Várias técnicas para a colocação de } \\
\text { CVC, como de marco anatômico, } \\
\text { ainda são válidas. Mas a orientação } \\
\text { por USG pode diminuir } \\
\text { complicações. Protocolos de } \\
\text { implantação/manutenção de CVC } \\
\text { são essenciais para redução de } \\
\text { agravos. }\end{array}$ \\
\hline $\begin{array}{l}\text { Telang, N et al }{ }^{20} \\
\text { Índia } \\
\text { PUBMED } \\
2017\end{array}$ & $\begin{array}{l}\text { Estimar a utilidade } \\
\text { diagnóstica do USG em } \\
\text { tempo real na detecção } \\
\text { de má posição da ponta } \\
\text { de linhas centrais }\end{array}$ & $\begin{array}{l}\text { Observacional, } \\
\text { piloto, } \\
\text { quantitativo }\end{array}$ & $\begin{array}{l}\text { Elegeu-se } 33 \text { pacientes para inserção do PICC. USG } \\
\text { e raio-X serviram para localizar a ponta do cateter } \\
\text { pós-inserção em } 21 \text { casos. Em } 10 \text { casos foi } \\
\text { necessário bolus de solução salina. Outros } 2 \text { foram } \\
\text { evidenciados em posição anômala. }\end{array}$ & $\begin{array}{l}\text { O estudo indicou valor preditivo } \\
\text { positivo para o uso de USG em } \\
\text { tempo real, podendo diminuir o } \\
\text { tempo necessário para o início das } \\
\text { infusões. }\end{array}$ \\
\hline $\begin{array}{l}\text { Johnson K et al }{ }^{21} \\
\text { Estados Unidos } \\
\text { PUBMED } \\
2016\end{array}$ & $\begin{array}{l}\text { Avaliar, de forma } \\
\text { retrospectiva, a } \\
\text { efetividade do uso de } \\
\text { USG na obtenção de } \\
\text { acesso venoso em } \\
\text { neonatos }<1,5 \mathrm{~kg} \text {, com } \\
\text { histórico de insucesso } \\
\text { prévio, em inserções, } \\
\text { realizadas por } \\
\text { enfermeiros. }\end{array}$ & $\begin{array}{l}\text { Observacional, de } \\
\text { coorte, } \\
\text { retrospectivo, } \\
\text { qualitativo }\end{array}$ & $\begin{array}{l}\text { Elencados } 10 \text { RN para uso do USG na tentativa de } \\
\text { estabelecer linha venosa, após diagnóstico de } \\
\text { enfermagem de acesso venoso difícil, mesmo com } \\
\text { uso de transiluminecência ou infravermelho. O } \\
\text { USG foi realizado por médicos. As veias preferidas } \\
\text { foram de membros superiores. Todas as punções } \\
\text { foram exitosas. Não houve complicações. }\end{array}$ & $\begin{array}{l}\text { O uso de USG para orientação de } \\
\text { inserção de cateter epicutâneo em } \\
\text { recém-nascidos de muito baixo peso } \\
\text { pode ser considerada técnica } \\
\text { efetiva, com baixo risco de } \\
\text { complicações, exigindo porém } \\
\text { treinamento e prática para uso } \\
\text { otimizado da aparelhagem. }\end{array}$ \\
\hline $\begin{array}{l}\text { Nguyen }{ }^{22} \\
\text { Estados Unidos } \\
\text { Google Scholar } \\
2016\end{array}$ & $\begin{array}{l}\text { Avaliar o benefício } \\
\text { clínico, considerações } \\
\text { práticas para } \\
\text { implementação e } \\
\text { limitações da USG para a } \\
\text { colocação de CVC } \\
\text { (umbilical e PICC) na } \\
\text { UTIN. }\end{array}$ & $\begin{array}{l}\text { Pesquisa } \\
\text { bibliográfica }\end{array}$ & $\begin{array}{l}\text { Cinco estudos sobre inserções de CVC, guiados por } \\
\text { USG, e sete estudos descrevendo USG pós- } \\
\text { inserção foram determinados como pertinentes ao } \\
\text { objetivo da revisão e discutidos. A literatura } \\
\text { pareceu insuficiente para recomendar USG como } \\
\text { substitutivo de raio-X para confirmar a ponta do } \\
\text { cateter. }\end{array}$ & $\begin{array}{l}\text { A utilização do USG durante a } \\
\text { inserção de CVC seguida de raio-X } \\
\text { tem potencial para reduzir } \\
\text { manipulações, necessidade de novo } \\
\text { raio-X e complicações. }\end{array}$ \\
\hline $\begin{array}{l}\text { Richter } R \text { et } a^{23} \\
\text { Estados Unidos } \\
\text { PUBMED } 2016\end{array}$ & $\begin{array}{l}\text { Descrever os resultados } \\
\text { de nova técnica de } \\
\text { cateterismo epicutâneo } \\
\text { por canulação da veia } \\
\text { femoral superficial distal } \\
\text { (VFSD), guiada por USG } \\
\text { em lactentes com } \\
\text { cardiopatia em UTIN. }\end{array}$ & $\begin{array}{l}\text { Coorte } \\
\text { retrospectivo, } \\
\text { Descritivo, } \\
\text { qualitativo }\end{array}$ & $\begin{array}{l}\text { Das } 31 \text { crianças submetidas à técnica, } 28 \\
\text { obtiveram êxito, sendo necessário o gasto de } 34 \\
\text { dispositivos ( } 82 \% \text { de sucesso). Não foram relatadas } \\
\text { taxas de punção arterial inadvertida. A taxa de } \\
\text { infecção associada à técnica foi de } 4,6 \% \text {. A taxa de } \\
\text { ocorrência de trombose venosa profunda foi de } \\
11,8 \% \text {. }\end{array}$ & $\begin{array}{l}\text { O uso da VFSD como primeira } \\
\text { escolha para inserção de PICC em } \\
\text { crianças com cardiopatia congênita } \\
\text { mostrou altas taxas de sucesso e } \\
\text { longevidade (de } 16 \text { a } 123 \text { dias, } \\
\text { média de } 39 \text { dias de permanência do } \\
\text { cateter), deixando livre a área } \\
\text { cardíaca para exploração cirúrgica. }\end{array}$ \\
\hline $\begin{array}{l}\text { Saul D et al }{ }^{24} \\
\text { Estados Unidos } \\
\text { PUBMED } \\
2016\end{array}$ & $\begin{array}{l}\text { Avaliar a efetividade do } \\
\text { USG, comparada ao raio- } \\
\text { X, para localização de } \\
\text { aparatos terapêuticos } \\
\text { (dentre eles cateter } \\
\text { umbilical e PICC). }\end{array}$ & $\begin{array}{l}\text { Estudo piloto, } \\
\text { prospectivo, } \\
\text { quantitativo. }\end{array}$ & $\begin{array}{l}\text { Trinta estudos de USG e raio-X foram realizados } \\
\text { em } 25 \text { pacientes, onde } 11 \text { foram submetidos à } \\
\text { cateterismo epicutâneo. Destes, } 10 \text { foram } \\
\text { visualizado, sendo que } 3 \text { estavam mal } \\
\text { posicionados. Em } 1 \text { caso não se visualizou a ponta } \\
\text { do cateter. }\end{array}$ & $\begin{array}{l}\text { USG foi efetiva para avaliação da } \\
\text { localização dos cateteres, resultado } \\
\text { que apoia a necessidade de mais } \\
\text { treinamento para adoção da USG } \\
\text { como rotina de avaliação de } \\
\text { dispositivos de suporte em terapia } \\
\text { intensiva. }\end{array}$ \\
\hline $\begin{array}{l}\text { Von-Jakitsch C et } \\
\text { al }^{25} \\
\text { Brasil } \\
\text { Google Scholar } \\
2016\end{array}$ & $\begin{array}{l}\text { Verificar nos } \\
\text { estabelecimentos de } \\
\text { saúde do Vale do } \\
\text { Paraíba paulista a } \\
\text { utilização do cateter } \\
\text { PICC e os profissionais } \\
\text { envolvidos no processo. }\end{array}$ & $\begin{array}{l}\text { Observacional, } \\
\text { descritivo, } \\
\text { quantitativo }\end{array}$ & $\begin{array}{l}\text { PICC era utilizado em } 70 \% \text { dos estabelecimentos } \\
\text { observados, com uso de protocolos nos processos. } \\
\text { O enfermeiro era responsável pela inserção e } \\
\text { remoção destes dispositivos. A minoria utilizava a } \\
\text { punção guiada por imagem para inserção. }\end{array}$ & $\begin{array}{l}\text { Nos cenários estudados, o PICC era } \\
\text { amplamente utilizado desde o ano } \\
\text { de } 2005 \text {. A competência do } \\
\text { enfermeiro habilitado era } \\
\text { reconhecida, sendo este o } \\
\text { responsável pela atuação diante } \\
\text { deste dispositivo. }\end{array}$ \\
\hline $\begin{array}{l}\text { YeX et al }^{26} \\
\text { Austrália } \\
\text { PUBMED } \\
2016\end{array}$ & $\begin{array}{l}\text { Fixar em um hospital } \\
\text { terciário, a probabilidade } \\
\text { de trombose venosa de } \\
\text { membro superior } \\
\text { (TVMS) em pacientes } \\
\text { com PICC ou CVC, versus } \\
\text { pacientes sem cateter. }\end{array}$ & $\begin{array}{l}\text { Retrospectivo, } \\
\text { quantitativo }\end{array}$ & $\begin{array}{l}\text { Das } 876 \text { USG, realizadas em } 637 \text { pacientes, } 213 \\
\text { evidenciaram TVMS. Em } 38 \text { (de 85) RN com PICC, } \\
\text { 36/103 dos pacientes com CVC, e em 139/688 dos } \\
\text { pacientes sem cateter in situ TVMS foi } \\
\text { diagnosticada. No período, } 1855 \text { PICC e } 2435 \text { CVC } \\
\text { foram inseridos no hospital. A taxa de incidência de } \\
\text { TVMS foi 2,05\% em pacientes com PICC e de 1,48\% } \\
\text { nos com CVC. }\end{array}$ & $\begin{array}{l}\text { Os cateteres predispõem à } \\
\text { formação de TVMS. Os PICC têm } \\
\text { maior probabilidade de estarem } \\
\text { associados à TVMS do que o CVC e } \\
\text { têm menos tempo para coágulo } \\
\text { sintomático. }\end{array}$ \\
\hline
\end{tabular}

Figura 4: Quadro Sinóptico das publicações referentes aos anos de 2016 e 2017. Niterói, RJ, Brasil, 2020.

Os autores tinham formação inicial em medicina, exceto uma enfermeira brasileira ${ }^{25}$. Quanto à abordagem, sete estudos eram quantitativos ${ }^{14,17,18,20,24-26}$ e oito qualitativos ${ }^{12,13,15,16,19,21-23}$. Entre os quantitativos, quatro observacionais, longitudinais, prospectivos ${ }^{14,17,18,20,24}$ (dois pilotos, cegados ${ }^{14,24}$ ), um observacional descritivo ${ }^{25}$ e um observacional retrospectivo ${ }^{26}$. Dos oito estudos qualitativos analisados, um era diretriz internacional ${ }^{12}$ e sete eram artigos (um relato de experiência ${ }^{13}$, um relato de caso ${ }^{16}$, dois de revisão de literatura ${ }^{19,22}$ e três retrospectivos, documentais ${ }^{15,21,23}$. 
Os estudos foram agrupados em dois eixos de aproximação temática: uso do USG na inserção do cateter PICC e uso do USG no diagnóstico de complicações relacionadas ao cateter. A maioria das publicações versavam sobre a utilização da USG em tempo real, à beira leito, possibilitada pelo advento das aparelhagens portáteis.

Em sete artigos originais, desenvolvidos com objetivo de avaliar o uso de ecografia portátil na técnica de inserção, o enfoque se distribuiu entre escolha do sítio de inserção ${ }^{21,23,25}$, e localização da ponta do cateter ${ }^{13,18,20,24}$. Já no documento contendo recomendação de especialistas ${ }^{12}$ e nas duas revisões de literatura ${ }^{19,22}$ discutiu-se o uso da ecografia para visualização venosa, progressão vascular e localização da ponta do cateter. Outros três artigos originais ${ }^{15,16,26}$ e uma dissertação ${ }^{17}$ avaliaram o uso da USG na fase de pós-inserção, para diagnóstico de complicações relacionadas ao PICC, como derrame pericárdico ${ }^{15}$, tamponamento cardíaco ${ }^{17}$, trombose venosa profunda ${ }^{26}$ e efusão pleural por nutrição parenteral ${ }^{16}$. Destes, um expôs a técnica denominada TNEcho (ecocardiografia neonatal direcionada) ${ }^{16}$ e outro não deixava claro o uso de aparelho portátil ${ }^{26}$.

Por fim, um estudo objetivou analisar a viabilidade de um protocolo de localização da ponta do cateter, quando utilizado por radiologistas ${ }^{14}$. E outro conhecer a utilização do PICC, os processos adotados e os profissionais envolvidos, em hospitais do interior paulista ${ }^{25}$.

\section{DISCUSSÃO}

A utilização do PICC em neonatologia consiste em uma prática especializada e preferencial, pela possibilidade de inserção à beira-leito, sem necessidade de intervenção cirúrgica, nem deslocamentos danosos ao RN instável; além disso, oferece menor risco para infecção de corrente sanguínea quando comparado ao cateter não tunelizado ${ }^{19,22}$

A USG alcançou grande aceitação e utilidade na prática clínica a partir do advento do aparecimento de aparelhos portáteis, que possibilitaram a técnica denominada POCUS ${ }^{12,15}$ (ultrassonografia realizada e interpretada à beira- leito), ou RTUS ${ }^{20}$ (ultrassom em tempo real, em inglês). Sua utilização difundiu-se entre profissionais médicos não radiologistas, que a incorporaram à sua prática diária, extrapolando os limites das especialidades. A técnica é defendida como útil para estabelecer diversos diagnósticos e vantajosa por não utilizar radiação ionizante, permitir estudos dinâmicos e não ser invasiva ${ }^{12,27}$.

No contexto da TIV, a adoção do POCUS em protocolos institucionais de inserção e manutenção do PICC estendeu a execução deste método de imagem ao enfermeiro, em países onde este profissional, é o responsável, junto com o médico, pela inserção deste dispositivo, o que se reflete nas normativas que regulam a atividade profissional $^{3}$. Porém apenas um estudo brasileiro, observacional evidenciou esta prática como rotineira, e de competência do enfermeiro, para orientar a venopunção, expondo que, mesmo previsto, o uso do POCUS ocorria na minoria das inserções ${ }^{25}$. No parecer de relator COFEN 243/2017, há análise de diversas normativas regionais que amparam o uso do USG, por enfermeiros habilitados no procedimento de inserção de PICC, limitado à visualização e escolha do vaso a ser puncionado, para otimização do introdutor, cateter e guia (para técnica de Seldinger modificada) $)^{3,28,29}$.

Um estudo estadunidense, de 2016, avaliou a utilização experimental de ecografia para inserção de PICC por profissionais médicos, em prematuros com peso menor que 1,5kg. As inserções ecoguiadas foram realizadas após diagnóstico de enfermagem de acesso venoso difícil, com $100 \%$ de êxito. Na instituição, USG não se aplicava em primeira escolha e os protocolos de enfermagem previam a seleção do vaso para inserção de PICC por técnica de palpação, uso de transiluminação ou infravermelho ${ }^{21}$, com taxa de sucesso superior a $90 \%$.

Em fevereiro de 2020, foi publicado um documento, elaborado por vinte especialistas, a maioria intensivista pediátrico ou neonatal, fruto de extensa pesquisa literária, e obedecendo a Avaliação Internacional de Diretrizes, Pesquisa e Avaliação (AGREE). Houve concordância em 39 das 41 indicações de uso do POCUS cardíaco, pulmonar, vascular, cerebral e abdominal em neonatos e crianças gravemente enfermos. Destas, 28 basearam-se em evidências de qualidade moderada ${ }^{12}$. Os autores concluíram que o POCUS é proficiente para inserção e localização da ponta do PICC. E consideraram suas recomendações úteis para o uso de POCUS nesta clientela e nortear programas de treinamento. Mas defenderam urgência em pesquisas adicionais, dada à fraca qualidade de evidências disponíveis nos estudos avaliados para a elaboração do guideline ${ }^{12}$. A maioria dos estudos discutidos nesta scoping review, observacionais, qualitativos, documentais, de literatura ou de campo, limitados por pequenas amostras, corroboram este achado, expondo evidências de nível baixo ${ }^{13,15,16,20,21,23,24}$.

Outro estudo, de revisão sistemática, divergiu, sustentando haver fortes evidências, oriundas de grandes estudos com metanálise, vinculando o uso de ecografia ao procedimento de PICC, desde a escolha do sítio venoso, passando pela progressão até a visualização da ponta do dispositivo, mostrando vantagens da USG em todos os processos de 
implantação. Os autores concluíram, inclusive, pela indicação de POCUS para PICC em RN de extremo baixo peso, por excelente taxa de êxito e baixo risco de complicações ${ }^{19}$.

Para a localização da ponta do cateter, indispensável para início da TIV, o exame de raios X ainda é o mais utilizado ${ }^{22}$. E com repetições, caso haja necessidade de reposicionamento do dispositivo venoso ${ }^{12,22}$. O que é desvantajoso, bem como a necessidade de rede vascular íntegra e calibrosa para inserção ${ }^{32}$. Autores defendem que o POCUS traz vantagens ao procedimento, como a localização exata da luz do vaso, mensuração precisa do seu diâmetro ${ }^{12,31}$ e visualização de veias profundas para estabelecimento PICC, como veia jugular interna (VJI) e femoral ${ }^{12,22,23}$. O uso do POCUS para visualizar VJI apresenta vantagens em relação à técnica por referência anatômica, como menor tempo de inserção, alto índice de sucesso na primeira tentativa e maior percentual da localização da ponta do dispositivo ${ }^{12,22}$.

Uma pesquisa bibliográfica abrangeu um estudo realizado por neonatologistas americanos experientes, que implantaram PICC com POCUS; como resultado, o tempo total do procedimento foi reduzido em $40 \%$. A necessidade de reposicionamento entre os cateteres implantados por USG e de manipulações adicionais foram menores quando comparado ao método padrão. Cinco de vinte lactentes (25\%) com PICC guiados por POCUS precisavam de ajustes na linha com base nos raios X em comparação com 19 dos 28 lactentes (68\%) na implantação padrão ${ }^{22}$.

Um coorte retrospectivo descreveu uma canulação da veia femoral superficial distal guiada por ultrassom em tempo real (DSFV) para PICC em lactente gravemente enfermos com cardiopatia congênita. A opção pelo uso da USG se deu por não haver pontos superficiais usados como referência para a identificação do acesso. Concluiu-se que, apesar das limitações do estudo, esta escolha de acesso obteve alta taxa de sucesso, suficiente longevidade e flexibilidade para TIV, necessitando de estudos prospectivos mais amplos no contexto de cuidados perioperatórios destes pacientes, bem como descrever as complicações associado a esta técnica ${ }^{23}$.

Tanta inovação tecnológica em TIV exige dos profissionais de UTIN empenho constante, a fim de renovar seus conhecimentos e desenvolver novas habilidades ${ }^{27}$. Um estudo inferiu que a experiência prática do médico seria essencial para executar com eficácia a escolha do sitio de punção, a partir de imagem ecográfica a beira leito. Mas não discutiu qual seria a quantidade de horas de treinamento e atividades práticas necessárias para chegar à expertise ${ }^{23}$. Outro estudo, brasileiro, sugeriu que as instituições implementassem programas de capacitação para enfermeiros, contribuindo para o aprimoramento destes profissionais e para segurança do paciente, já que evidências científicas correlacionam o uso desta tecnologia ao aumento do sucesso de implantação em rede venosa difícil ${ }^{25}$.

\section{CONCLUSÃO}

A POCUS é uma inovação promissora para uso em terapia intensiva neonatal. A miniaturização das máquinas de USG, com manutenção da qualidade de imagem e redução de custos, pavimentou o caminho para o uso desta técnica em procedimentos diagnósticos diversos, além do uso para inserção e manutenção do PICC. O que transforma a POCUS em prática com alta relação custo-efetividade, suportado agora por opinião de especialistas internacionais.

A tendência é que a POCUS seja adotada como primeira escolha para eleição da veia a ser canulizada. E como método alternativo para guiar a inserção do PICC, por possibilidade técnica (visto que, para realização destas duas etapas do processo de inserção com uso de USG, seria necessário o uso de um único transdutor, sem inclusão de outros materiais), o que legitimará o profissional enfermeiro como habilitado para técnica em todo o mundo. Porém, estudos recentes não excluem a radiografia de controle da posição da ponta do PICC, indicando a realização das duas técnicas, comparativamente, apontando o POCUS como suplementar ao exame radiológico. Estudos futuros, de maior impacto, podem sustentar o valor preditivo positivo do POCUS.

Como limitação do processo desta revisão de escopo apontamos a escolha de três bases de dados que disponibilizam estudos na área da saúde do Brasil, América Latina e EUA em mais 70 países. O que pode ter excluído estudos publicados em diversos países, com possíveis resultados importantes para a temática. Cabe também ressaltar a fragilidade dos próprios estudos incluídos nesta scoping review. Segundo exposto por alguns autores, muitos resultados obtidos, necessitariam ser suportados por outros estudos, com amostras maiores, para sua generalização.

\section{REFERÊNCIAS}

1. Lopes MR. Complicações relacionadas ao uso de cateter central de inserção periférica em UTI Neonatal no Brasil. [Graduação em Enfermagem] Niterói: Universidade Federal Fluminense, 2014.

2. Di Santo MK, Takemoto D, Nascimento RG, Nascimento AM, Siqueira, Duarte $C T$ et al. Peripherally inserted central venous catheters: alternative or first choice vascular cited? J. vasc. bras. [Internet]. 2017 [cited 2020 Mar 16]; 16(2):104-12. DOI: http://dx.doi.org/10.1590/1677-5449.011516

3. Conselho Federal de Enfermagem. Parecer de Relator COFEN 243/2017. Atualiza a normatização do procedimento de inserção, fixação, manutenção e retirada de cateter periférico central por Enfermeiro - PICC. 2017 [cited 2020 Mar 16]; Available from: http://www.cofen.gov.br/parecer-de-relator-cofen-no-2432017_57604.html 
4. Shalabi M, Adel M, Yoon E, Khalid A, Shoo L, Prakesh SS. Risk of infection using peripherally inserted central and umbilical catheters in preterm neonates. Pediatrics [Internet]. 2015 [cited 2020 Mar 16]; 136:1073-9. DOI: https://doi.org/10.1542/peds.2015-2710

5. Duesing LA, Fawley JA, Wagner AJ. Central venous cited in the pediatric population with emphasis on complications and prevention strategies. Nutr. Clin. Pract. [Internet] 2016 [cited 2020 Mar 16]; 31:490-501. Available from: https://doi.org/10.1177/0884533616640454

6. Lacerda MAS, Silva T, Khoury HJ, Vieira JNM, Matushita JPK. Risks of radiographic procedures for neonates admitted to a public hospital in Belo Horizonte, MG, Brazil. Radiol. Bras. [Internet]. 2008 [cited 2020 Apr 14]; 41(5):325-329. Available from: http://dx.doi.org/10.1590/S0100-39842008000500011

7. Kaji T, Kawano T, Yamada W, Yamada K, Onishi S, Nakame $\mathrm{K}$ et al. The changing profile of safe techniques for the insertion of a central venous catheter in pediatric patients - improvement in the outcome with the experiences of 500 insertions in a single institution. J. Pediatr. Surg. [Internet] 2016 December [cited 2020 Apr 14]; 51(12):2044-2047. Available from: https://doi.org/10.1016/j.jpedsurg.2016.09.037

8. Infusion Nurses Society. Infusion nursing standards of practice. J. Infus. Nurs. [Internet] 2006 [cited 2020 Apr 14]; 29(1Suppl):S1-S92. DOI: https://doi.org/10.1097/00129804-200601001-00001

9. Peters MDJ, Godfrey CM, McInerney P, Soares CB, Khalil H, Parker D. The Joanna Briggs Institute reviewers' manual 2015: methodology for JBI scoping reviews [Internet]. 2015 [cited 2020 Apr 14]; Available from: https://nursing.Isuhsc.edu/JBI/docs/ReviewersManuals/Scoping-.pdf

10. Aromataris E, Munn Z (Editores). Manual do Revisor do Instituto Joanna Briggs. The Joanna Briggs Institute. 2017 [cited 2020 Mar 16]; Available from: https://reviewersmanual.joannabriggs.org/

11. Arksey H, O'Malley L. Scoping studies: towards a methodological framework, International Journal of Social Research Methodology, [Internet] 2005 [cited 2020 mar. 16]; 8(1):19-32. Available from: https://www.tandfonline.com/doi/abs/10.1080/1364557032000119616

12. Singh Y, Tissot C, Fraga MV, Yousef N, Cortes RG, Lopez J, et al. International evidence-based guidelines on Point of Care Ultrasound (POCUS) for critically ill neonates and children issued by the POCUS Working Group of the European Society of Paediatric and Neonatal Intensive Care (ESPNIC). Crit. Care [Internet] 2020 [cited 2020 Feb 24]; 24(1):65. Available from: https://www.repository.cam.ac.uk/handle/1810/303870

13. Suell JV, Meshkati M, Juliano C, Groves A. Real-time point-of-care ultrasound-guided correction of PICC line placement by external manipulation of the upper extremity. Arch Dis Child Fetal Neonatal Ed. [Internet] 2020 [cited 2020 Apr 14]; 105 (1):25. DOI: http://dx.doi.org/10.1136/archdischild-2019-317610

14. Motz P, Arnim AVSAV, Likes M, Chabra S, Traudt C, lyer RS, et al. Limited ultrasound protocol for upper extremity peripherally inserted central catheter monitoring: a pilot study in the neonatal intensive care unit. J. Ultrasound Med. [Internet] 2019 [cited 2020 Apr 24]; 38(5):1341-1347. Available from: https://www.ncbi.nlm.nih.gov/pubmed/30244492

15. Barreiros LL, Andrade FM, Torres RA, Magalhães LVB, Farnetano BS, Fiorelli RKA. Cardiac tamponade by peripherally inserted central catheter in preterm infants: role of bedside ultrasonography and therapeutic approach. Rev. Col. Bras. Cir. [Internet] 2018 [cited 2020 Apr 15]; 45(3):e1818. Available from: http://www.scielo.br/scielo.php?pid=S0100-69912018000300157 \&script=sci_arttext\&tIng=en

16. Sancak S, Tuten A, Yildirim TG, Karatekin G. Massive pleural effusion on the contralateral side of a venous peripherally inserted central catheter. J. Clin. Ultrasound. [Internet] 2018 [cited 2020 Mar 15]; 46(2):140-4. Available from: https://www.onlinelibrary.wiley.com/doi/abs/10.1002/jcu.22493

17. Torres RA. A prática e as perspectivas da ultrassonografia point-of-care em unidade de terapia intensiva neonatal. [PósGraduação em Ciência da Saúde]. Viçosa (MG): Universidade Federal de Viçosa, 2018.

18. Zaghloul N, Watkins L, Choi-Rosen J, Perveen S, Kurepa D. The superiority of point of care ultrasound in localizing central venous line tip position over time. Eur. J. Pediatr. [Internet] 2019 [cited 2020 Apr 25]; 178(2):173-9. Available from: https://www.ncbi.nlm.nih.gov/pubmed/30374753

19. Ares G, Hunter CJ. Central venous cited in children: indications, devices, and risks. Curr. Opin. Pediatr. [Internet] 2017 [cited 2020 Mar 25]; 29(3):340-6. Available from: https://www.ncbi.nlm.nih.gov/pubmed/28323667

20. Telang N, Sharma D, Pratap OT, Kandraju H, Murki S. Use of real-time ultrasound for locating tip position in neonates undergoing peripherally inserted central catheter insertion: a pilot study. Indian. J. Med. Res. [Internet] 2017 Mar [cited 2020 Mar 25]; 145(3):373-376. Available from: https://www.ncbi.nlm.nih.gov/pubmed/28749401

21. Johnson KN, Thomas T, Grove J, Jarboe MD. Insertion of peripherally inserted central catheters in neonates less than $1.5 \mathrm{~kg}$ using ultrasound guidance. Pediatr. Surg. Int. [Internet] 2016 [cited 2020 Apr 19]; 32(11):1053-7. Available from: https://www.ncbi.nlm.nih.gov/pubmed/27590475

22. Nguyen J. Ultrasonography for central catheter placement in the neonatal intensive care unit-a review of utility and practicality. Am J. Perinatol. [Internet] 2016 [cited 2020 Mar 25]; 33(6):525-30. DOI: https://doi.org/10.1055/s-0035-1569987

23. Richter RP, Law MA, Borasino S, Surd JA, Alten JA. Distal Superficial Femoral Vein Cannulation for Peripherally Inserted Central Catheter Placement in Infants with Cardiac Disease. Congenit Heart Dis. [Internet] 2016 Dec [cited 2020 Mar 25]; 11(6):733-740. Available from: https://doi.org/10.1111/chd.12398

24. Saul D, Ajayi S, Schutzman DL, Horrow MM. Sonography for Complete Evaluation of Neonatal Intensive Care Unit Central Support Devices: A Pilot Study. J. Ultrasound Med. [Internet] 2016 Jul [cited 2020 Apr. 14]; 35(7):1465-73. DOI: https://doi.org/10.7863/ultra.15.06104 
25. Von-Jakitsch CB, Carvalho DPL, Posso MMS, Machado RCM, Giaretta VMA. Peripherally inserted central catheter: the use in Vale do Paraíba Paulista. Rev. Enferm. UFPE on line. 2016 [cited 2020 Mar 14]; 8(2):4280-9. Available from: http://www.seer.unirio.br/index.php/cuidadofundamental/article/view/4354

26. Ye X, Wong SW, Zhang J, Moo IH, Lee CC. Catheter-related upper limb venous thrombosis in a tertiary hospital setting. ANZ J. Surg. [Internet] 2016 Dec [cited 2020 Mar 25]; 86(12):1033-7. DOI: https://doi.org/10.1111/ans.12865

27. Bastos M, Vieira ALS, Pazeli Jr JM. Uso da ultrassonografia "point-of-care" na prática nefrológica: Transpondo os limites do trato urinário. Revista HU [Internet]. 2019 [cited 2020 Apr 06]; 45(3):341-5. Available from: https://periodicos.ufff.br/index.php/hurevista/article/view/28745

28. Bortoli PS, Leite ACAB, Alvarenga WA, Alvarenga CS, Bessa CR, Nascimento LC. Peripherally inserted central catheter in pediatric oncology: a scoping review. Acta paul. enferm. [Internet] 2019 [cited 2020 Apr 05]; 32(2):220-228. DOI: http://dx.doi.org/10.1590/1982-0194201900030

29. Alcântara DC, Peregrino AAF, Jesus CS, Siqueira AP, Silva PO, Marta CB et al. Peripherally inserted central catheter: contributions to oncological nursing. Rev. enferm. UFPE on line. 2019 [cited 2020 Apr 05]; 13(3):715-31. Available from: https://periodicos.ufpe.br/revistas/revistaenfermagem/article/view/236058/31568

30. Oliveira CR, Neve ET, Rodrigues EC, Zamberlan KC, Silveira A. Cateter central de inserção periférica em pediatria e neonatologia: possibilidades de sistematização em hospital universitário. Escola Anna Nery Revista de Enfermagem [Internet]. 2014 [cited 2020 Apr 15]; 18(3):379-85. Available from: http://www.scielo.br/pdf/ean/v18n3/1414-8145-ean-18-03-0379.pdf

31. Infusion Nurses Society Brasil - INS Brasil. Diretrizes Práticas para Terapia Infusional. 94 páginas. Ano 2013. 